\title{
AVERAGE SURVIVAL OF PATIENTS TREATED ON STAGE I AND CLINICALLY NO BREAST CANCER, FROM JANUARY 2010 TO DECEMBER 2019 SUBMITTED TO AXILLARY LYMPHONODECTOMY OR SENTINEL LYMPH NODE
}

Anna Lya Menezes Martins ${ }^{1}$, André Mattar ${ }^{1}$, Felipe Andreotta Cavagna , Luiz Henrique Gebrim¹ ${ }^{1}$ Hospital Pérola Byington - São Paulo (SP), Brazil.

Introduction: Axillary lymph node involvement is an important assessment in breast cancer staging, prognosis and predicts therapeutic specificity. Despite the large proportion of advanced cases in the Public Health System in Brazil, about $67 \%$ of our patients are in stages I and II. The absence of reports of survival in Brazil motivated us to carry out this study. Objectives: To retrospectively assess the median survival of 2,170 stage I breast cancer patients who underwent axillary lymph nodes dissection (ALND) compared to those who underwent only sentinel lymph node biopsy (SLNB) using the patent blue technique. Methods: This is a cross-sectional, retrospective clinical study, aiming to assess the average survival of patients with stage I breast cancer without clinical lymph node involvement (N0), submitted to ALND after positive SLNB during the surgical procedure or SLNB failure guided by patent blue. 2,170 patients were included in stage I. The study was carried out between January 2010 and December 2019. Results: The average number of sentinel lymph nodes was 3.2. In patients in stage I, the mean survival for more than five years was higher in those submitted only to a $98.31 \%$ SLNB ( $\mathrm{n}=1228$ ). In those who performed ALND, survival was $94.13 \%(\mathrm{n}=867)$. In less than five years, longer survival was observed in patients who underwent ALND 5.87\% $(\mathrm{n}=54)$. Axillary lymph node dissection was essential in the treatment of breast cancer for many years in the last group. However, high morbidity directly interfered with the patients' quality of life. Therefore, long-term studies introduced SLNB as a standard for axillary evaluation in clinically T1, T2 and N0 patients, noting that patients with negative SLNB and not submitted to ALND showed no difference in axillary recurrence, disease-free survival and overall survival. Our results at Stage I, although the 5-year period is short, overlap with those in the literature. Over the years, surgical axillary staging and management of early breast cancer have evolved, becoming less invasive and more conservative, with imaging exams playing an important role in axillary disease, with the use of improved techniques in an attempt to minimize intervention. Conclusions: In view of the data presented, we concluded that the average survival for more than five years was higher in patients who underwent only $98.31 \%$ SLNB compared to those who underwent ALND (94.13\%). 